\title{
Orbital Polarons in the Metal-Insulator Transition of Manganites
}

\author{
R. Kilian and G. Khaliullin \\ Max-Planck-Institut für Physik komplexer Systeme, Nöthnitzer Strasse 38, D-01187 Dresden, Germany
}

(15 March, 1999)

\begin{abstract}
The metal-insulator transition in manganites is strongly influenced by the concentration of holes present in the system. Based upon an orbitally degenerate Mott-Hubbard model we analyze two possible localization scenarios to account for this doping dependence: First, we rule out that the transition is initiated by a disorder-order crossover in the orbital sector, showing that its effect on charge mobility is only small. Second, we introduce the idea of orbital polarons originating from a strong polarization of orbitals in the vicinity of holes. Considering this direct coupling between charge and orbital degree of freedom in addition to lattice effects we are able to explain well the phase diagram of manganites for low and intermediate hole concentrations.
\end{abstract}

PACS number(s): 72.80.Ga, 71.30.+h, 71.38.+i, 75.50.Cc

The doping dependence of the properties of manganites, $R_{1-x} A_{x} \mathrm{MnO}_{3}$ (where $R$ and $A$ represent rare-earth and divalent metal ions, respectively), poses some of the most interesting open problems in the physics of these compounds. First to be noticed is the peculiar asymmetry of the phase diagram that is most pronounced in the charge sector: Regions of high $(x>0.5)$ and low $(x<0.5)$ concentration of holes are characterized by such contrasting phenomena as charge ordering and metalicity, respectively. In the latter region - which we wish to focus on - the metallic state can be turned into an insulating one by raising the temperature above $T_{C} \approx 200$ - $350 \mathrm{~K}$. Introducing the notion of double exchange [1] which associates the relative orientation of localized Mn $t_{2 g}$ spins with the mobility of itinerant $e_{g}$ electron, early work has identified this transition to be controlled by the loss of ferromagnetic order inherent to the metallic state. It is believed that the lattice effects are also of crucial importance in this transition. Within the lattice polaron - double exchange picture [2], the crossover from metallic to insulating behavior is controlled by the ratio of polaron binding energy to the kinetic energy of charge carriers. Assuming the former quantity to be constant, a critical coupling strength leading to localization can be reached by reducing the kinetic energy, i.e., by raising temperature which acts via the double-exchange mechanism. Spin disorder and spin polaron effects [3] further enhance the carrier localization above $T_{C}$. The doping dependence of the metal-insulator transition, however, is not fully captured in this picture. Namely the complete breakdown of metalicity at hole concentrations below $x_{\text {crit }} \approx 0.15-0.2$ that occurs despite the fact that the fully saturated ferromagnetic state is sustained remains an open problem which we will address in this paper.

We analyze two mechanisms that could drive the localization of doped holes at small $x$. First, we explore the possibility of the metal-insulator transition to be controlled by a disorder-order crossover in the $e_{g}$-orbital sector. We outrule this scenario by showing that the mobility of doped holes depends only little on the degree of order in the orbital background. Second, we introduce the concept of orbital polarons in an orbitally degenerate Mott-Hubbard system - this constitutes the main idea of the paper. Similar to spin polarons in correlated spin systems such as cuprates, the orbital polaron is a natural consequence of strong correlations and the double degeneracy of on-site levels, the latter being a consequence of the $e_{g}$-orbital degeneracy present in ferromagnetic manganites. We argue that the coupling between charge carriers and orbital degrees of freedom yields a strong polarization of $e_{g}$ orbitals in the neighborhood of holes that acts together with breathing-mode like lattice distortions to form combined orbital-lattice polarons. The effect is more pronounced at small $x$ because the polarizability of orbitals enhances as the characteristic energy scale of orbital fluctuations $\propto x t$ reduces. Within the orbitallattice polaron scenario we are able to explain well the phase diagram of manganites for low and intermediate doping levels.

Orbital order-disorder and hole mobility. - As the frustrating effect of holes is weakened at small $x$, a JahnTeller- and superexchange-driven transition from a disordered, strongly fluctuating orbital state to an orbitally ordered state is expected. This possibly suggests the localization process to be triggered by the establishment of order in the orbital sector, which would naturally introduce a doping dependence due to the energy scale $x t$ of orbital fluctuations. We analyze this possibility by comparing the mobility of holes moving in orbitally disordered and ordered backgrounds. The relevant transfer Hamiltonian of $e_{g}$ electrons has to account for strong onsite Coulomb repulsions and the orbital degeneracy:

$$
H_{t}=-\sum_{\langle i j\rangle_{\gamma}} \sum_{\alpha \beta}\left(t_{\gamma}^{\alpha \beta} \hat{c}_{i \alpha}^{\dagger} \hat{c}_{j \beta}+\text { H.c. }\right)
$$

with $\gamma \in\{x, y, z\}$. We use constrained operators $\hat{c}_{i \alpha}^{\dagger}=$ $c_{i \alpha}^{\dagger}\left(1-n_{i}\right)$ that create electrons at site $i$ in orbital $\alpha$ only under the condition that the site is empty. In orbital basis $\alpha \in\left\{\left|3 z^{2}-r^{2}\right\rangle,\left|x^{2}-y^{2}\right\rangle\right\}$ the intersite transfer matrices are: 


$$
t_{x / y}^{\alpha \beta}=t\left(\begin{array}{cc}
1 / 4 & \mp \sqrt{3} / 4 \\
\mp \sqrt{3} / 4 & 3 / 4
\end{array}\right), \quad t_{z}^{\alpha \beta}=t\left(\begin{array}{ll}
1 & 0 \\
0 & 0
\end{array}\right) .
$$

Electron spins are treated within the double-exchange model and implicitly enter through the transfer amplitude $t$ only. To observe the strongly correlated nature of Hamiltonian (11) it is convenient to introduce separate quasiparticles for charge and orbital degrees of freedom [4. In the case of an orbitally disordered state we employ a slave-boson representation in which electrons are replaced by fermionic orbitons $f_{i \alpha}^{\dagger}$ carrying orbital pseudospin and bosonic holons $b_{i}^{\dagger}$ carrying charge [5]. On a mean-field level with parameters $\sum_{\alpha \beta} t_{\gamma}^{\alpha \beta}\left\langle f_{i \alpha}^{\dagger} f_{j \beta}\right\rangle_{\gamma}=\chi$ and $\left\langle b_{i}^{\dagger}\right\rangle=\sqrt{x}$ these quasiparticles can be decoupled. The characteristic energy scale of orbital fluctuations is then set by the orbiton half band width $D_{\text {orb }}=3 x t$, the mobility of holes by the holon half band width $D_{\text {hole }}=3 t$.

Orbital order in manganites is induced by a coupling of orbital pseudospins mediated by, e.g., Jahn-Teller phonons and superexchange. The corresponding Hamiltonian (see, e.g., [5.,6]) is of $X Y$-type with internal frustration making it difficult to handle. To simplify the discussion we simulate an orbitally ordered state by adding to Hamiltonian (11) the interaction term

$$
H_{J}=-\frac{J}{2 z} \sum_{\langle i j\rangle_{\gamma}} \sigma_{i}^{x} \sigma_{j}^{x} e^{i q_{\gamma}}
$$

with $z=6$ and Pauli matrices $\sigma_{i}^{x}$. For $\boldsymbol{q}=(\pi, \pi, 0)$ Eq. (2) favors a staggered $\left(\left|3 z^{2}-r^{2}\right\rangle \pm\left|x^{2}-y^{2}\right\rangle\right) / \sqrt{2}$ ordering within $x-y$ planes repeating itself along the $z$ direction; this closely resembles the type of ordering observed experimentally in $\mathrm{LaMnO}_{3}$ []]. The establishment of orbital order is indicated by a singularity in the static susceptibility $\left\langle\sigma^{x} \sigma^{x}\right\rangle_{\boldsymbol{q}}$. In random-phase approximation

$$
\left\langle\sigma^{x} \sigma^{x}\right\rangle_{\boldsymbol{q}}=\frac{\left\langle\sigma^{x} \sigma^{x}\right\rangle_{\boldsymbol{q}}^{0}}{1+J_{\boldsymbol{q}}\left\langle\sigma^{x} \sigma^{x}\right\rangle_{\boldsymbol{q}}^{0} / 2}
$$

with $J_{q}=J\left(\cos q_{x}+\cos q_{y}-\cos q_{z}\right) / 3$. Bare susceptibilities $\langle\cdots\rangle^{0}$ are treated employing the above meanfield description. Numerically solving for the pole of Eq. (3) with $J=0.13 \mathrm{eV}$ as estimated from the structural phase transition observed in $\mathrm{LaMnO}_{3}$ at $T=780 \mathrm{~K}$ [7] and $t=0.4 \mathrm{eV}$ we find a critical doping concentration $x_{\text {crit }}=J / 4 t=0.08$; at concentrations below this critical value an orbitally ordered state is to be expected.

We now turn to analyze the effect of orbital order onto the mobility of holes. For this we study the evolution of the holon half band width, which in the disordered state $D_{\text {hole }}=3 t$, as orbital order develops. Foremost, an important difference between models with orbital pseudospin and conventional spin is to be noticed: In the latter systems spin is conserved when electrons are transferred between sites. In contrast, the transfer Hamiltonian (11) of the orbital model is nondiagonal in orbital pseudospin - the transfer matrices $t_{x}^{\alpha \beta}, t_{y}^{\alpha \beta}$, and $t_{z}^{\alpha \beta}$ cannot be diagonalized simultaneously. Hence there is a finite amplitude for holes to hop without perturbing the orbitally ordered background, leading to the formation of a wide coherent hole band; for the specific type of order introduced above its half width is $D_{\text {hole }}^{\text {coh }}=2 t$. This number seems to indicate a profound reduction in the mobility of charge carriers by $\approx 30 \%$ as compared to the disordered state. It does, however, not reflect the full kinetic energy of holes which is further contributed to by incoherent processes involving the creation of excitations in the orbital background. To study the influence of these processes we employ an "orbital-wave" approximation that uses a bosonic description of orbital excitations of energy $J$ and a fermionic description of holons moving within a band of half width $2 t$. Holons are coupled to orbital excitations via part of Hamiltonian (1). In analogy to studies of spin systems [8] we analyze the motion of a single hole employing a self-consistent Born approximation. Two limits can be treated analytically: As orbital excitation become very heavy, $J / t \rightarrow \infty$, the hole decouples from the orbital subsector. It then moves coherently due to the nondiagonality of transfer matrices alone, its motion being maximally constrained as $D_{\text {hole }}=2 t$. In the opposite limit, $J / t \rightarrow 0$, the hole moves completely incoherently in a cloud of an infinite number of orbital excitations suppressing orbital correlations in the background. The hole mobility then reaches its maximum $D_{\text {hole }}=3 t$ as in the disordered state, while the effective mass of the composite object formed of hole and excitation cloud becomes large, $m \rightarrow \infty$. For a ratio $J / t=0.3$ realistic for manganites one is closer to the latter limit: Numerically we find the establishment of orbital order to reduce the kinetic energy of the hole by only less than $5 \%$. We therefore conclude that a disorder-order crossover in the orbital sector has only a secondary effect on the mobility of charge carriers, outruling it as a driving mechanism to initiate the metal-insulator transition.

Orbital polarons . - In the preceding paragraph we have considered charge carriers and orbitals to interact via the transfer part (11) of the Hamiltonian. We will now show that in an orbitally degenerate Mott-Hubbard system there also exists a direct coupling stemming from a strong polarization of $e_{g}$ orbitals on sites next to a hole. The lifting of orbital degeneracy is mediated by a displacement of oxygen atoms situated between the Mn sites as well as by the $e_{g}$ level splitting in the vicinity of positively charged holes. We estimate the magnitude of the degeneracy lifting, $\Delta=\Delta^{\mathrm{ph}}+\Delta^{\mathrm{ch}}$, as follows: Holes respectively electrons are coupled to the lattice breathing mode $Q_{1}$ and to two Jahn-Teller modes $Q_{2}$ and $Q_{3}$ by

$$
\begin{aligned}
H_{\mathrm{el-ph}}= & \sum_{i}\left(-g_{1} Q_{1, i} n_{i}^{h}+g_{2}\left(Q_{2, i} \sigma_{i}^{x}+Q_{3, i} \sigma_{i}^{z}\right)\right. \\
& \left.+\frac{K}{2} \boldsymbol{Q}_{i}^{2}\right),
\end{aligned}
$$


where $n_{i}^{h}$ is the number operators for holes. Integrating over oxygen displacements $\boldsymbol{Q}=\left(Q_{1}, Q_{2}, Q_{3}\right)$ we obtain $\Delta^{\mathrm{ph}}=g_{1} g_{2} \sqrt{2} /(3 K) \approx\left(g_{1} / g_{2}\right) E_{\mathrm{JT}}$. A lower bound for this quantity is given by the Jahn-Teller energy $E_{\mathrm{JT}} \approx 0.1-0.2 \mathrm{eV}$, assuming that coupling to the breathing mode is at least as strong as to the Jahn-Teller one. On the other hand, the contribution to the $e_{g}$ level splitting from the Coulomb interaction between the positively charged hole and an electron on a neighboring site is estimated taking into account the covalency of Mn $3 d$ and $\mathrm{O} 2 p$ orbitals. It follows $\Delta^{\text {ch }} \approx \frac{3}{4} \gamma^{2} e^{2} R_{\mathrm{Mn}-\mathrm{Mn}}$, where the covalency factor $\gamma=t_{p d} / \Delta_{p d}$ can be obtained from the transfer amplitude and the charge gap between $\mathrm{Mn}$ and $\mathrm{O}$ sites, $t_{p d} \approx 1.8 \mathrm{eV}$ and $\Delta_{p d} \approx 4.5 \mathrm{eV}$ [9], respectively. With lattice spacing $R_{\mathrm{Mn}-\mathrm{Mn}}=3.9 \AA$ this leads to $\Delta^{\text {ch }} \approx 0.4 \mathrm{eV}$. In total, the polarization of $e_{g}$ levels on sites next to a hole yields an energy splitting $\Delta \approx 0.5$ - $0.6 \mathrm{eV}$. Being comparable in magnitude to the transfer amplitude $t$ this number strongly indicates a direct coupling of charge and orbital degrees of freedom to be of importance in manganites.

In the cubic system the Hamiltonian describing the above coupling is

$$
H_{\mathrm{ch}-\mathrm{orb}}=-\Delta \sum_{\langle i j\rangle_{\gamma}} n_{i}^{h} \tau_{j}^{\gamma}
$$

where $\tau_{j}^{\gamma}=\left(\sin \Theta \sigma^{x}+\cos \Theta \sigma^{z}\right) / 2$ denotes orbital pseudospin operators with angles $\Theta=\mp 2 \pi / 3,0$ corresponding to bonds in directions $\gamma=x, y, z$. Hamiltonian (5) promotes the formation of orbital polarons. For low enough hole concentrations these consist of a bound state between a central hole and the surrounding $e_{g}$ orbitals pointing towards the hole. Besides minimizing interaction energy this configuration yields a large amplitude of virtual excursions of $e_{g}$ electrons onto the empty site, thus lowering the kinetic energy as well. In other words, the double exchange process is locally enhanced, providing a large effective spin of the orbital polaron. This naturally explains the development of ferromagnetic clusters experimentally observed at temperature above $T_{C}$ [10]. At large doping concentrations, orbital polarons cannot be considered as isolated anymore. In the presence of many holes, the fast fluctuations of orbitals destroy the orbital-hole bound state. To elaborate on this point we calculate the orbital-hole binding energy $E_{\text {orb }}$ by considering a static hole in an orbital background. The upper bound for $E_{\text {orb }}$ is set by the limiting case of uncorrelated, static orbitals: Hamiltonian (5) yields $E_{\text {orb }}=3 \Delta(1-x)$ if all six orbitals surrounding a hole point towards the latter, where the factor $(1-x)$ accounts for the fact that some of the orbitals might be empty. In the real system, however, the binding energy is reduced by inter-orbital coupling with energy scale $\propto J$ and orbital fluctuations of frequency $\propto x t$ which are comprised in the effective "stiffness" of the orbital sector $D_{\text {orb }}=3 x t+J$. Calculating the binding energy for a static hole in both orbitally ordered and disordered states we find an interpolating formula

$$
E_{\text {orb }}=3\left(\sqrt{\Delta^{2}(1-x)^{2}+D_{\text {orb }}^{2}}-D_{\text {orb }}\right),
$$

which correctly reproduces the limits $\Delta \gg D_{\text {orb }}$ and $\Delta \ll D_{\text {orb }}$. In marked contrast to conventional lattice polaron theory, the binding energy of the orbital polaron explicitly depends on the concentration of holes, foremost via the energy scale of orbital fluctuations $x t$ : The more holes are present in the system the faster orbitals fluctuate and the smaller the binding energy $E_{\text {orb }}$ becomes. The crossover from the small-polaron regime in which the orbital-hole bound state is stable to the large-polaron regime in which the bound state breaks up is governed by the dimensionless coupling constant $\lambda_{\text {orb }}=E_{\text {orb }} / D_{\text {hole }}$, where $D_{\text {hole }}$ is the half band width of holes. Since the lattice breathing mode in Eq. (4) further enhances the polaron formation, the total coupling constant $\lambda=\lambda_{\text {orb }}+\lambda_{\text {ph }}$ has an additional contribution $\lambda_{\mathrm{ph}}=E_{\mathrm{ph}} / D_{\text {hole }}$, where $E_{\mathrm{ph}}=g_{1}^{2} /(2 K)$. It is important to note that a critical value of $\lambda$ leading to localization can be reached either by increasing temperature which quenches $D_{\text {hole via double exchange or by lowering }}$ $x$ which enhances $E_{\text {orb }}$.

In the following we study in more detail the consequences of the polarization of orbitals around doped holes for the phase diagram of manganites. We start from the strong-coupling limit $\lambda \gg 1$ in which holes are substituted for by small polarons that move in a band whose width is reduced by an exponential factor

$$
e^{-\eta}=\exp \left[-\gamma\left(\frac{E_{\text {orb }}}{D_{\text {orb }}}+\frac{E_{\mathrm{ph}}}{\omega_{0}}\right)\right] \text {, }
$$

where $\omega_{0}$ is the frequency of dispersionless phonons. In deriving Eq. (7) we neglect the interference between orbital and lattice couplings. To simulate the crossover from the small polaron to the free carrier picture we phenomenologically employ the function $\gamma=\beta-$ $\ln [\lambda(1+\beta)] / \lambda^{2}$ with $\beta=\left(1-1 / \lambda^{2}\right)^{1 / 2}$ which has been proposed for strongly coupled phonon systems [11]; for $\lambda<1$ holes are assumed to move freely as $\gamma=0$. A feedback of Eq. (7) into the binding energy, Eq. (6), resulting from the quenching of orbital fluctuations $\propto x t e^{-\eta}$ as small polarons begin to form sharpens the transition. Next we turn to the coupling between the charge and spin sector through the double-exchange mechanism. On the one hand, the transfer amplitude $t=t_{0}\left[\left(1+m^{2}\right) / 2\right]^{1 / 2}$ depends on the magnetization $m$. This is determined from $m=B_{S}(\alpha m)$ with $\alpha=3 S /(1+S) T_{C} / T$, where $B_{S}$ is the Brillouin function and $S=\frac{3}{2}+(1-x) \frac{1}{2}$ the average spin of Mn $3 d$ electrons per site. On the other hand, charge transfer mediates a ferromagnetic interaction between sites. The effective exchange coupling constant is 


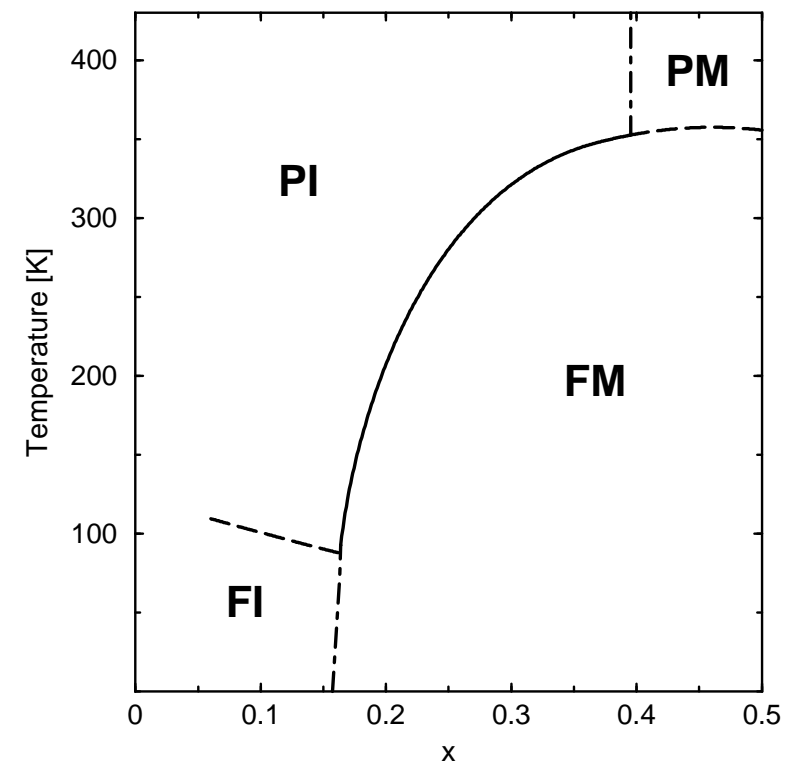

FIG. 1. Magnetic and electronic phase diagram obtained within the present theory. Dashed and dashed-dotted lines represent magnetic and electronic transitions, respectively, the simultaneous transition in both channels is denoted by a solid line. The phases are: paramagnetic insulator (PI), paramagnetic metal (PM), ferromagnetic metal (FM), and ferromagnetic insulator (FI).

$$
J_{\text {eff }}=\frac{t \chi}{2 S^{2}}\left[x(1-x) e^{-\eta}+x_{0}(1-x)^{2}\right]
$$

with $x_{0}=2 t \chi / U$, where $U$ is the on-site repulsion, and mean-field parameter $\chi \approx \frac{1}{2}$. The Curie temperature is obtained from $T_{C}=\frac{1}{3} \nu z \stackrel{S}{S}(S+1) J_{\text {eff }}$; the fitting parameter $\nu$ compensates for the overestimation of $T_{C}$ in mean-field treatment. The first term in squared brackets of Eq. (8) stems from the coherent motion of polarons and represents the conventional double-exchange contribution to $T_{C}$. The second term is due to superexchange. It is insensitive to the localization process and dominates ferromagnetic interaction at low doping levels 12 14.

To illustrate the interplay between the system of equations (6) - (8) we numerically extract from them the $T-x$ phase diagram. The following parameters are chosen: The orbital polarization energy is set to $\Delta=0.5 \mathrm{eV}$, yielding a binding energy comparable to the phononic one $E_{\mathrm{ph}}=0.6 \mathrm{eV}$; the phonon frequency is $\omega_{0}=0.05 \mathrm{eV}$, the interaction between orbitals $J=0.13 \mathrm{eV}$, the bare transfer amplitude $t_{0}=0.4 \mathrm{eV}$, and $x_{0}=0.1$. The fitting parameter $\nu=0.5[15]$ is adjusted to reproduce the values of $T_{C}$ observed for $\mathrm{La}_{1-x} \mathrm{Sr}_{x} \mathrm{MnO}_{3}$ [16]. The result is shown in Fig. 1. Due to the doping dependence of the orbital polaron binding energy the system is more insulating at low and more metallic at high doping levels. This is manifested in the absence of a metallic phase at doping levels $x<0.15$ and the appearance of a paramagnetic metallic phase at $x>0.4$. In the region in between a simultaneous magnetic and electronic transition from a ferromagnetic metal to a paramagnetic insulator occurs. We note that the loss of charge mobility triggers static orbital order. A long-range orbitally ordered state has in fact been experimentally detected in the insulating regions of $\mathrm{La}_{0.88} \mathrm{Sr}_{0.12} \mathrm{MnO}_{3}$ [13]. In general, however, the ordered state is expected to have orbital and Jahn-Teller glass features due to the presence of quenched orbital polarons. The orbital polaron provides a strong local ferromagnetic coupling between the planes in c-direction, thus explaining the existence of the isotropic ferromagnetism even in the insulating phase at small doping. Finally it is worth to notice that the phase diagram in this theory is highly sensitive to the transfer amplitude $t_{0}$ as this parameter enters in the polaron binding energy, Eq. (5).

We conclude that orbital polarons are an intrinsic part of an orbitally degenerate Mott-Hubbard system and play an important role in the physics of manganites. Being comparable in magnitude to lattice effects this new mechanism naturally introduces a doping dependence into the polaron binding energy via the degree of orbital fluctuations. Accounting for both orbital and lattice effects we are able to reproduce well the important features of the phase diagram of manganites.

[1] C. Zener, Phys. Rev. 82, 403 (1951); P. W. Anderson and H. Hasegawa, ibid. 100, 675 (1955); P.-G. de Gennes, ibid. 118, 141 (1960).

[2] A. J. Millis, P. B. Littlewood, and B. I. Shraiman, Phys. Rev. Lett. 74, 5144 (1995); A. J. Millis, Nature (London) 392, 147 (1998).

[3] C. M. Varma, Phys. Rev. B 54, 7328 (1996).

[4] S. Ishihara, M. Yamanaka, and N. Nagaosa, Phys. Rev. B 56, 686 (1997).

[5] R. Kilian and G. Khaliullin, Phys. Rev. B 58, R11 841 (1998).

[6] S. Ishihara, J. Inoue, and S. Maekawa, Phys. Rev. B 55, 8280 (1997).

[7] Y. Murakami et al., Phys. Rev. Lett. 81, 582 (1998).

[8] C. L. Kane, P. A. Lee, and N. Read, Phys. Rev. B 39, 6880 (1989).

[9] T. Saitoh et al., Phys. Rev. B 51, 13942 (1995).

[10] J. M. Teresa et al., Nature (London) 386, 256 (1997).

[11] A. S. Alexandrov, V. V. Kabanov, and D. K. Ray, Phys. Rev. B 49, 9915 (1994).

[12] R. Maezono, S. Ishihara, and N. Nagaosa, Phys. Rev. B 58, 11583 (1998).

[13] Y. Endoh et al. (unpublished).

[14] G. Khaliullin, R. Kilian (unpublished).

[15] This factor can partly be attributed to the fluctuation correction $\nu=0.7$ to mean-field $T_{C}$ which follows from Eq. (5.4) in G. S. Rushbrooke, G. A. Baker, and P. J. Wood, in Phase Transitions and Critical Phenomena, edited by C. Domb and M. S. Green (Academic, New 
York, 1974).

[16] A. Urushibara et al., Phys. Rev. B 51, 14103 (1995). 